\title{
Écologie et distribution de l'abeille originaire d'Asie Megachile sculpturalis SMITH I853 (Apoidea - Megachilidae - Megachilini) : un état des connaissances dix ans après sa première observation en Europe
}

parViolette LE FÉON* et Benoît GESLIN***

Résumé. Megachile sculpturalis est une abeille originaire de l'est de l'Asie observée pour la première fois en Europe en 2008 à Allauch près de Marseille (France). Elle a également été détectée aux ÉtatsUnis dès 1994 et s'est depuis largement répandue en Amérique du Nord. Nous présentons ici un état des connaissances sur son écologie et sa distribution géographique.

Abstract. Megachile sculpturalis (the Giant Resin Bee) is a bee species native to East Asia that has been observed for the first time in Europe in 2008 in Allauch near Marseille (France). In the USA, it has been reported in 1994 and has since then rapidly expanded through North America. We here provide a review on its ecology and its geographical distribution.

Mots-clés : Megachile sculpturalis, distribution, espèce exotique, préférences alimentaires, nidification

Keywords : Megachile sculpturalis, distribution, exotic species, diet preferences, nesting behaviour

\section{Introduction}

Sur le continent américain et en Océanie, de nombreuses espèces d'abeilles exotiques (souvent originaires d'Europe) ont été observées (GOULSON 2003 ; RUSSO 2016). Leur présence hors de leur aire d'origine résulte d'introductions volontaires (par exemple Bombus terrestris (L. 1758) et Megachile rotundata (FABRICIUS 1793), espèces introduites pour la pollinisation et Apis mellifera L. 1758, introduite pour la pollinisation et la production de miel) ou involontaires. Dans le nord-est des États-Unis par exemple, BARTOMEUs et al. (2013) ont recensé 20 espèces

\footnotetext{
* Observatoire des Abeilles, 2 domaine Bellevue, I I 290 Arzens, France - violette.lefeon@gmail.com

** Aix Marseille Univ, Univ Avignon, CNRS, IRD, IMBE, AixMarseille Université - Campus Étoile Faculté des Sciences StJérôme Case 421 av Escadrille Normandie Niémen 13397 Marseille Cedex 20, France - benoit.geslin@gmail.com
}

d'abeilles exotiques et leur nombre ne cesse de croître (GIBBS \& DATHE 2017).

En Europe, les abeilles d'origine exotique sont plus rares. En 1996, une espèce nord-américaine, Xylocopa virginica (L. 1771), a été observée à quelques reprises en Angleterre (FALK \& LEWINGTON 2015). Dans leur récente mise à jour de la liste des abeilles d'Europe, RASMONT et al. (2017) citent plusieurs espèces du genre Xylocopa originaires d'Afrique ou du Proche-Orient récemment observées en Europe mais excepté pour $X$. pubescens SPINOLA 1838, bien établie en Grèce, les observations correspondantes ne semblent que ponctuelles. Enfin, Megachile disjunctiformis COCKERELL 1911, originaire de l'est de l'Asie, a été ponctuellement observée (5 spécimens au total) dans l'agglomération de Bologne en Italie en 2011, 2016 et 2017 (BorTolotTI et al. 2018). 
Megachile (Callomegachile) sculpturalis SMITH 1853, également originaire de l'est de l'Asie, est la seule espèce d'abeille introduite connue pour s'être installée dans plusieurs pays d'Europe. La première observation a été réalisée en 2008 dans le sud de la France (Vereecken \& Barbier 2009). Nous présentons ici un état des connaissances sur la distribution géographique et l'écologie de M. sculpturalis.

\section{Description}

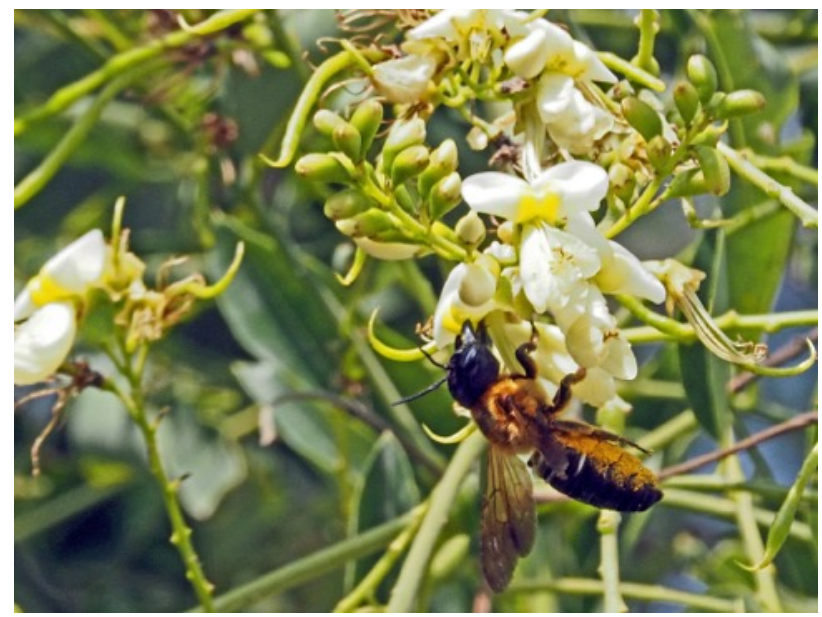

Figure I. Femelle de Megachile sculpturalis sur une fleur de Sophora japonica.

La brosse ventrale est recouverte de pollen.

Danièle Tixier-Inrep, Bouillargues (Gard), France, 2014.

M. sculpturalis est une mégachile de grande taille, les femelles mesurant de 22 à $27 \mathrm{~mm}$ de long et les mâles entre 14 et $19 \mathrm{~mm}$ (BATRA 1998). En France, rares sont les espèces dépassant deux centimètres de long : Xylocopa violacea (L. 1758), X. valga GERSTÄCKER 1872 et les reines de certains bourdons. Les individus des deux sexes ont des ailes fumées, un thorax recouvert de poils roux et un abdomen noir. Les femelles possèdent une brosse de poils (ou scopa) sous l'abdomen pour la récolte du pollen (fig. 1). Outre l'absence de brosse ventrale, les mâles se reconnaissent à la frange de poils clairs sur le clypéus, formant une sorte de moustache (fig. 2).

M. sculpturalis est facile à observer de par sa grande taille et son comportement (notamment car elle utilise fréquemment les hôtels à abeilles). Sous réserve de quelques précautions de rigueur (d'autres abeilles, des andrènes notamment, ont aussi un thorax roux et un abdomen noir), ses caractéristiques morphologiques en font une espèce facilement identifiable sur le terrain ou à partir d'une photographie. Elle ne peut normalement être confondue avec d'autres espèces d'abeilles en Europe, y compris avec l'abeille asiatique observée en Italie $M$. disjunctiformis qui est noire et blanche (BoRTOLOTTI et al. 2018). Le suivi de sa distribution géographique par un large réseau d'observateurs (sur le modèle des sciences participatives), même débutant dans l'étude des abeilles sauvages, est donc possible.

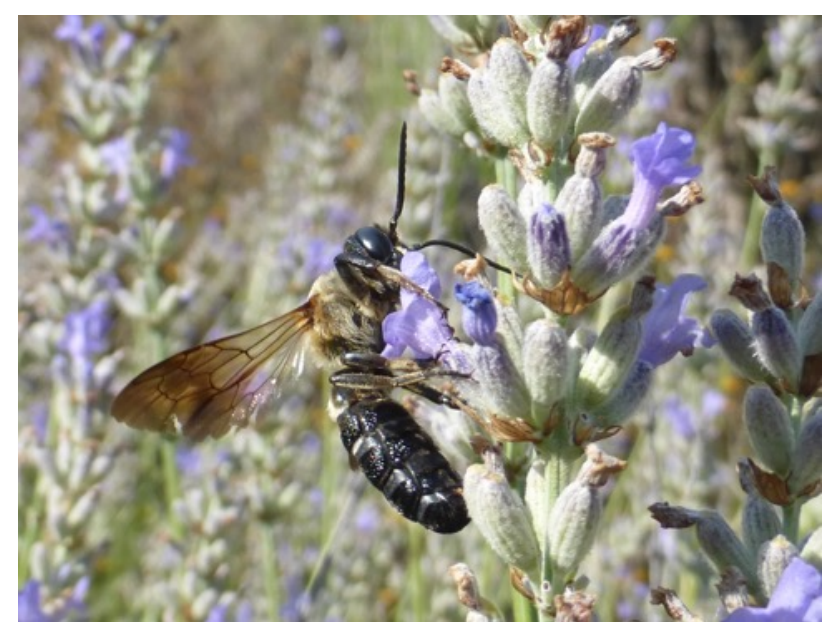

Figure 2. Mâle de Megachile sculpturalis consommant du nectar sur une fleur de lavande (Lavandula sp.).

Gilles Roux, Saint-Antonin-sur-Bayon

(Bouches-du-Rhône), France, 2016.

\section{Distribution géographique}

L'aire d'origine de $M$. sculpturalis comprend plusieurs pays de l'est de l'Asie : la Chine, le Japon, Taïwan et la péninsule coréenne (Hinojosa-DíAZ et al. 2005). C'est par ailleurs désormais une espèce exotique en Amérique du Nord et en Europe. Elle a été observée aux ÉtatsUnis (État de Caroline du Nord) dès 1994 (MANGUM \& BROOK 1997) et s'est depuis largement répandue (MANGUM \& SUMNER 2003 ; HiNOJOSA-DÍAZ 2008 ; PARYS et al. 2015). Elle occupe aujourd'hui presque toute la moitié est du pays, la limite ouest de répartition étant atteinte dans le Texas et la limite sud en Floride (PARYs et al. 2015). Elle est également présente au Canada (PAIERO \& BUCK 2003).

En Europe, M. sculpturalis a été observée pour la première fois en 2008 en France métropolitaine, à Allauch (13) près de la ville portuaire de Marseille (VEREECKEN \& BARBIER 2009). Elle a ensuite été observée en Italie en 2009 (QUARANTA et al. 2014), en Suisse près de la frontière italo-suisse en 2010 (AMIET 2012) puis au nord des Alpes en 2015 (DiLliER 2016), en Allemagne en 2015 (WESTRICH et al. 2015), en 
Hongrie en 2015 (Kovács 2015) et en Autriche en 2017 (P. Westrich, com. pers.) (fig. 3). La chronologie des découvertes ne reflète cependant pas nécessairement la chronologie réelle de son expansion. Par ailleurs, en l'absence d'analyses génétiques, il n'est pas établi si ces différentes populations sont issues d'une introduction unique (et le cas échéant si celle-ci a eu lieu en France) ou s'il y en a eu plusieurs (voir ZAYED $e t$ al. 2007 pour un exemple de ce type d'étude chez les abeilles).

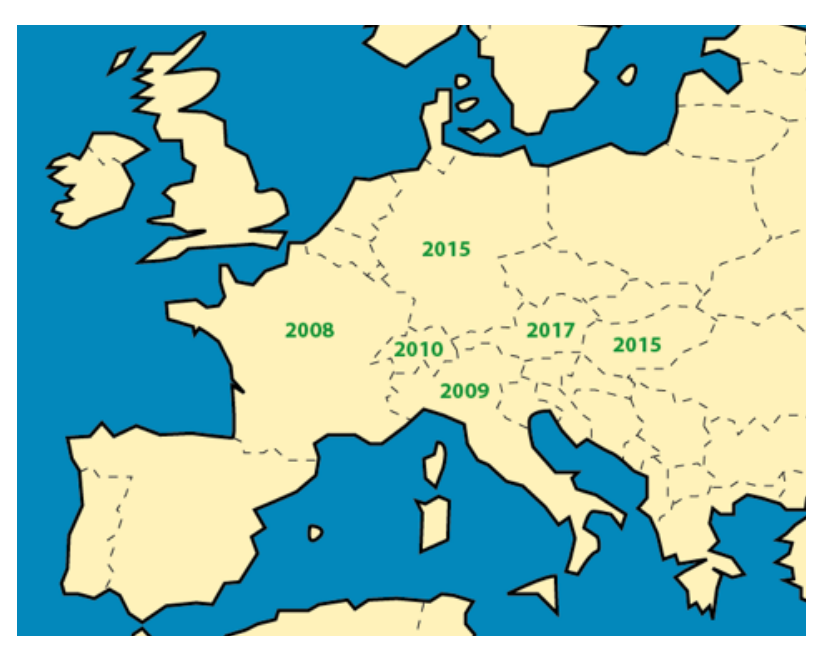

Figure 3. Année de première observation de Megachile sculpturalis dans les pays européens (Sources : Amiet 2012 ; Kovács 2015 ; Quaranta et al. 2014 ; Vereecken \& Barbier 2009 ; Westrich et al. 2015 ; Westrich, com. pers.).

En France, M. sculpturalis semble avoir rapidement étendu son aire de distribution depuis sa détection en 2008 (fig. 4). Entre 2008 et 2016, elle a été observée dans 72 communes, principalement dans le sud-est du pays, au sud de Lyon (69) et à l'est de Montpellier (34) (LE FÉON et al. 2018). Les observations ont eu lieu aussi bien au cœur de grandes villes que dans des zones rurales, et à une altitude maximale de 1540 m (un mâle observé à Matemale dans les Pyrénées-Orientales en 2013). En 2016, une nette progression vers l'ouest a été constatée (avec des observations à Toulouse (31), en Dordogne et dans les Pyrénées-Atlantiques à 520 $\mathrm{km}$ d'Allauch) et le nord (une femelle observée à Mâcon (71) à $335 \mathrm{~km}$ d'Allauch). En 2017², la présence de l'espèce dans l'ouest du pays se confirme avec une nouvelle observation aux environs de Toulouse (commune de Saint-Orens-
de-Gameville (31), 1 mâle, le 12 août 2017, par Hélène Laviron ${ }^{2}$ ) et dans le département des Landes (commune de Saint-Sever (40), 1 femelle, le 2 septembre 2017, par Philippe Dauge ${ }^{3}$ ) (fig. 4). En 2017, nous enregistrons également deux nouvelles observations en altitude, cette fois dans les Alpes-de-Haute-Provence (commune d'Allos (04), 1 mâle, le 2 août 2017, altitude = 1550 m, par Matthieu Aubert ; commune de Larche (04), 1 mâle, le 6 juillet 2017, altitude = 1846 m, par Eric Dufrêne) ainsi qu'une observation dans une région où l'espèce est déjà bien présente (commune de Saou (26), 1 individu, le 2 juillet 2017, par ascalaf074).

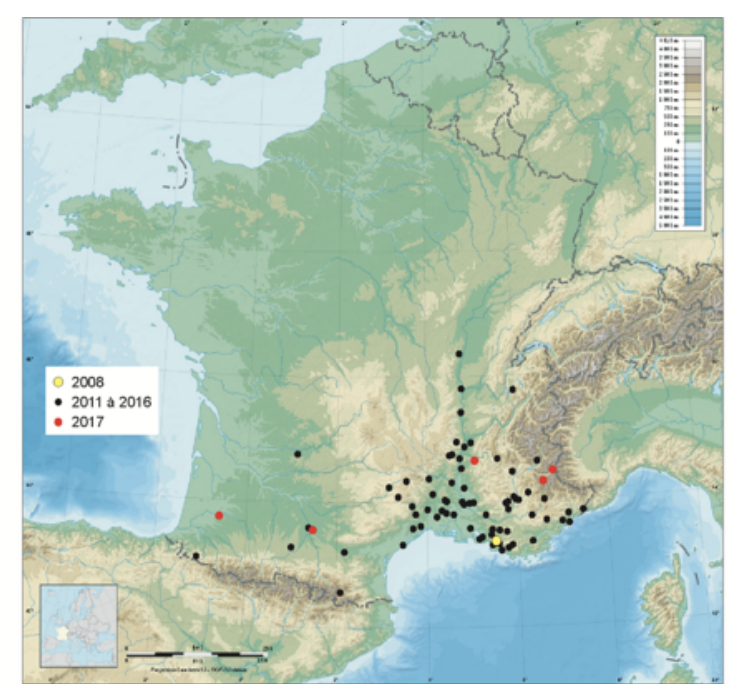

Figure 4. Distribution géographique de Megachile sculpturalis en France. Chaque point représente une commune. Le point jaune indique Allauch, lieu de la première observation de l'espèce en France, en 2008. Les points noirs indiquent les communes où une ou plusieurs observations ont eu lieu entre 2011 et 2016 (nous n'avons pas connaissance d'observations en 2009 et 2010). Les points rouges figurent les cinq communes mentionnées dans le texte, dans lesquelles la première observation a eu lieu en 2017.

Cette rapide expansion résulte probablement à la fois d'une composante naturelle (forte capacité de vol de l'espèce liée à sa grande taille) et d'une composante anthropique (déplacements d'adultes ou de larves par les échanges commerciaux, de bois notamment). Anthidium manicatum (L. 1758) (STRANGE et al. 2011) et Bombus terrestris (GESLIN \& MORALES 2015) sont deux autres exemples d'abeilles ayant rapidement étendu

\footnotetext{
I Nous mentionnons dans les lignes qui suivent les observations réalisées en 2017 constituant une première mention pour l'espèce dans la commune concernée.

${ }^{2}$ https://www.insecte.org/forum/viewtopic.php?f=3\&t=173263\&hilit=megachile+sculpturalis

3 https://www.flickr.com/photos/51044789@N02/369977I2745

${ }^{4}$ http://www.spipoll.org/galeries
} 
leur aire de distribution (respectivement en Amérique du Nord et en Patagonie) suite à une introduction hors de leur aire d'origine.

\section{Comportement alimentaire}

La nourriture exclusive des abeilles est constituée de ressources tirées des fleurs : le pollen (source de protéines), le nectar (source de sucres) et parfois des huiles florales (MICHENER 2007). Les adultes, mâles et femelles, consomment les ressources pour leurs besoins propres. De plus, les femelles récoltent les ressources (pollen principalement) pour les larves (exception faite des abeilles-coucous).

Le nombre de familles de plantes sur lequel une espèce d'abeille collecte du pollen détermine sa spécialisation alimentaire. Plusieurs auteurs ont proposé des classifications élaborées tenant compte de la diversité des comportements alimentaires (par exemple CANE \& SIPES 2006 ; Müller \& Kuhlmann 2008). Selon la classification simplifiée habituellement retenue, on qualifie d'oligolectique une espèce collectant le pollen sur des plantes d'une seule famille et de polylectique une espèce collectant le pollen sur des plantes de plusieurs familles (cf. par exemple NiETO et al. 2014). Le nectar est généralement collecté sur de nombreuses espèces végétales, y compris chez les abeilles oligolectiques.

Concernant le comportement alimentaire de $M$. sculpturalis dans son aire d'origine, BATRA (1998) indique qu'au Japon, le kudzu (Pueraria lobata = Pueraria hirsuta (THUNB.) C.K.SCHNEID. 1907, Fabacées) est sa source de pollen préférentielle, sans toutefois qu'elle ne soit exclusive, le même auteur citant plusieurs autres plantes. Pour la Chine, la plante citée est Lagerstroemia indica L. 1759, un arbuste de la famille des Lythracées.

Les observations réalisées aux États-Unis et en Europe indiquent que $M$. sculpturalis visite de nombreuses espèces végétales, de différentes familles, pour s'alimenter. Ainsi, dans leur synthèse de la littérature, PARYs et al. (2015) listent plus de 40 espèces végétales visitées, pour 21 familles. En France, entre 2008 et 2016, M. sculpturalis a été observée sur 20 espèces végétales appartenant à 8 familles différentes. 6 d'entre elles sont originaires d'Asie et ont été introduites comme plantes ornementales. Les autres sont des plantes indigènes (LE FÉON et al.
2018). Les plantes les plus fréquemment visitées étaient les lavandes (Lavandula spp., famille des Lamiacées) et l'arbre ornemental originaire d'Asie, Sophora japonica L. 1767 (famille des Fabacées). Les lavandes étaient la plante la plus visitée par les mâles, ce qui indique que c'est, au moins, une source de nectar préférentielle. Sophora japonica était la plante la plus visitée par les femelles.

Les observations de visites florales à elles seules ne permettent pas de statuer sur la spécialisation alimentaire d'une espèce d'abeille. L'analyse du contenu pollinique des scopa des femelles ou des cellules larvaires est nécessaire pour connaitre les espèces sur lesquelles le pollen est collecté et donc le statut de l'espèce, oligolectique ou polylectique. A notre connaissance, seuls sont disponibles les résultats d'analyses polliniques de quelques échantillons européens. En Italie, QUARANTA et al. (2014) ont trouvé que le contenu d'une cellule larvaire était composé de $81 \%$ de pollen de troène (genre Ligustrum, famille des Oléacées) et de $12 \%$ de pollen de châtaigner (genre Castanea, famille des Fagacées), d'autres pollens étant présents à l'état de traces. En Europe, les genres Ligustrum et Castanea comprennent une espèce indigène (respectivement L. vulgare L. 1753 et C. sativa Mill. 1768) et plusieurs espèces ornementales, notamment originaires d'Asie. Les résultats de QUARANTA et al. (2014) ne permettent pas de déterminer la nature du pollen au-delà du niveau générique et de conclure quant à l'origine européenne ou introduite des plantes utilisées.

WESTRICH et al. (2015) mentionnent le cas d'une cellule larvaire trouvée en Allemagne et de plusieurs cellules larvaires collectées en France ne contenant que du pollen de Sophora japonica. ANDRIEU-PONEL et al. (2018) ont analysé le pollen collecté sur plusieurs femelles à Nîmes et ont trouvé exclusivement du pollen de Sophora. Par ailleurs, le pollen d'une cellule larvaire collectée dans un hôtel à insectes à Montpellier était constitué de $97 \%$ de pollen de Sophora, les $3 \%$ restant provenant d'une trentaine d'espèces différentes (ANDRIEU-PONEL et al. 2018). Ces résultats posent la question des pollens présents en très petites quantités : proviennent-ils des récoltes de la femelle ou de résidus présents avant son installation dans cette cavité ?

Les analyses polliniques indiquent que $M$. sculpturalis collecte du pollen sur des plantes de 
différentes familles et peut donc être qualifiée de polylectique. Ces analyses sont cependant trop peu nombreuses pour conclure quant au niveau d'affinité de l'espèce avec certaines plantes et en particulier si elle dépend de la présence de plantes d'origine asiatique. L'espèce semble montrer une préférence pour $S$. japonica ou pour le genre Ligustrum mais dans quelles mesures ces résultats sont-ils généralisables ? C'est une question importante car l'approfondissement des connaissances permettra de déterminer si l'expansion de $M$. sculpturalis peut être limitée en l'absence de ces plantes nourricières et, parallèlement, si l'utilisation croissante de certaines plantes ornementales, telle que $S$. japonica, pourrait la favoriser.

\section{Nidification}

Comme de nombreuses espèces de Megachilidae, M. sculpturalis utilise des cavités préexistantes pour nidifier. Autrement dit, elle ne creuse pas elle-même, contrairement aux femelles de xylocopes par exemple, qui sont capables de creuser une cavité dans le bois. M. sculpturalis utilise divers types de cavités : " tiges " de bambou ou de canne de Provence, trous dans le bois mort (bûches) ou les vieux arbres, trous dans les poutres des maisons. Les cellules larvaires sont construites en résine de conifères ou d'érable par exemple (BATRA 1998), d'où son nom anglais de " Giant Resin Bee ». Les parois du nid, autour des cellules en résine, comportent de petites quantités de terre. Le bouchon terminal (qui sépare le nid de l'extérieur) est constitué de terre et parfois garni de débris divers, tels que des petits morceaux de bois, de paille, de coton (BATRA 1998) ou des pétales de fleurs (fig. 5). Sur les 39 cas de nidification recensés en France entre 2008 et 2016 (LE FÉON et al. 2018), les deux tiers concernent un hôtel à abeilles, avec dans certains cas l'emploi de tiges, dans d'autres de bûches percées. Pour le reste, la nidification a eu lieu dans des vieux arbres ou dans une poutre. Ce constat est peut-être un artefact associé à la plus forte présence d'observateurs aux abords des hôtels à abeilles (en général situés dans des lieux de passage : jardins, parcs) plutôt qu'autour des vieux arbres mais ce résultat témoigne d'une utilisation fréquente des hôtels à abeilles. Dans une étude initiée dans les parcs et jardins de la ville de Marseille, il est apparu que $M$. sculpturalis utilise massivement les hôtels à abeilles dès leur première année d'installation (B. Geslin, obs. pers.). Lors d'une étude menée sur trois ans dans la ville canadienne de Toronto, MACIVOR \& PACKER (2015) ont trouvé que les spécimens d'abeilles exotiques (parmi lesquels M. sculpturalis) représentaient $47,1 \%$ des abeilles collectées dans les hôtels à abeilles.

Dès les premières observations de $M$. sculpturalis aux États-Unis, l'utilisation de cavités précédemment utilisées par des xylocopes (X. virginica) a été constatée (MANGUM \& BROOK 1997) mais on ignorait si les femelles étaient venues occuper ces cavités une fois les occupants initiaux partis ou si celles-ci pouvait chasser les occupants pour s'installer dans leurs nids. Quelques années plus tard, LAPORT \& MINCKLEY (2012) ainsi que Roulston \& MALFI (2012) ont observé des femelles de $M$. sculpturalis chassant de leur nid des femelles de $X$. virginica puis vidant le contenu de la cavité afin d'y installer leurs propres cellules larvaires. Ce type de comportement avait déjà été observé chez une espèce européenne introduite aux États-Unis, Megachile apicalis SPINOLA 1808 (BARTHELL \& THORP 1995 in BARTHELL et al. 1998).

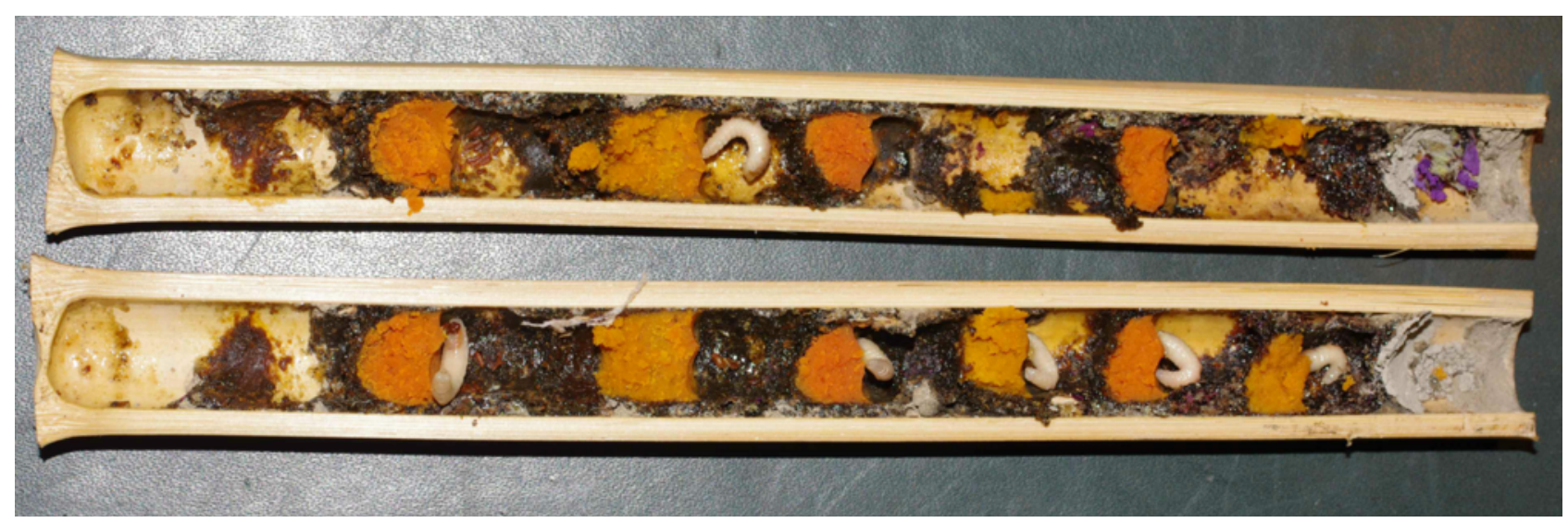

Figure 5. Tubes de bambous sectionnés pour observer la nidification de Megachile sculpturalis. Plusieurs cellules larvaires sont visibles, contenant une réserve de pollen et, parfois, une larve. Les cellules sont séparées les unes des autres par de la résine. Le bouchon terminal est constitué de terre et de pétales de fleurs (dans le bambou du haut). Daniel Mathieu,Avignon (Vaucluse), France, 2014. 


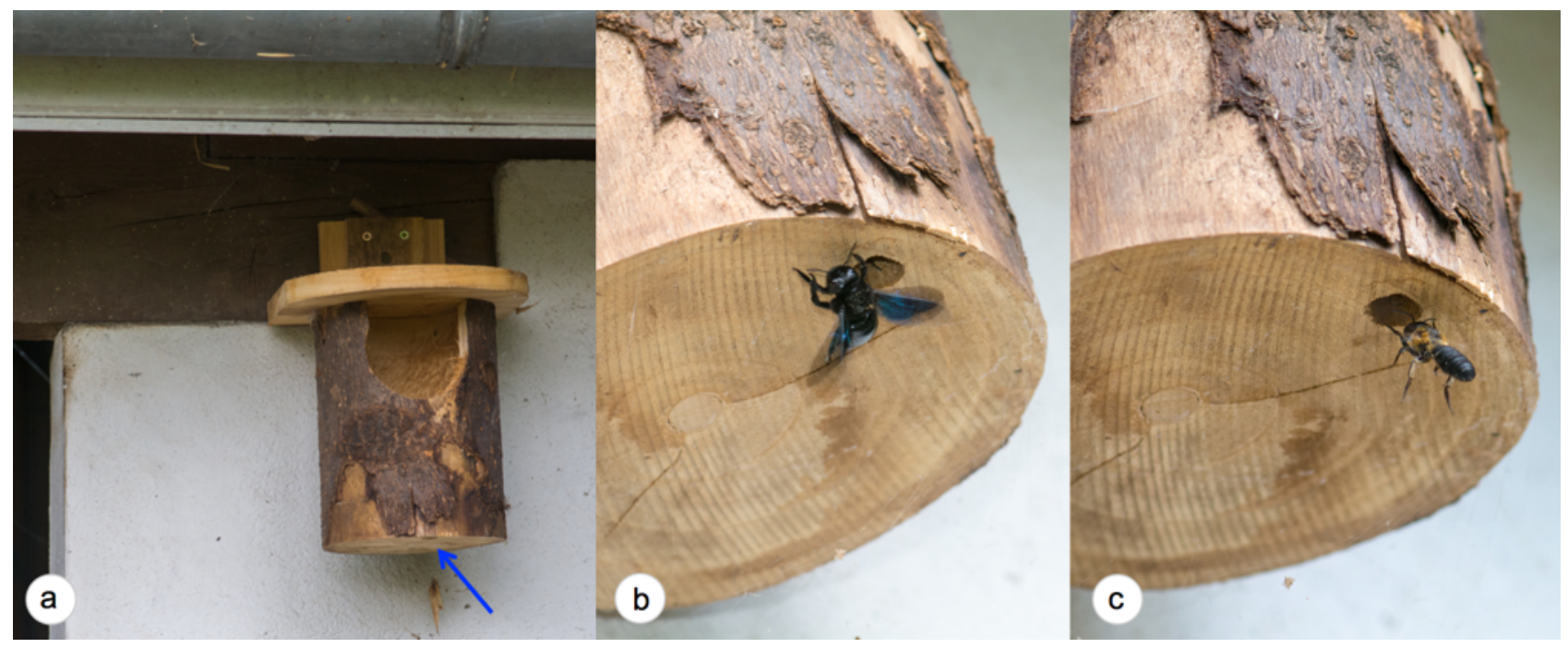

Figure 6. Trou dans une bûche (flèche bleue sur l'image a) dans lequel une femelle de Xylocopa violacea (b) et une femelle de Megachile sculpturalis (c) font des allers et venues au cours de la même journée.

Romain Baghi, Toulouse (Haute-Garonne), France, 2016.

En France, l'utilisation de cavités, dans du bois, précédemment occupées (et souvent creusées) par des xylocopes a été observée dans 5 communes différentes, selon les témoignages que nous avons recueillis. Des femelles de $X$. violacea et $M$. sculpturalis visitant la même cavité au cours de la même journée ont été observées (fig. 6) mais sans qu'un comportement agressif ou qu'un " vol » de nid (retrait du contenu d'un nid par M. sculpturalis pour y installer le sien) n'ait été constaté. Une cohabitation serait-elle possible au sein de mêmes cavités à l'image des cohabitations entre espèces du genre Xylocopa et Lithurgus (DUFRÊNE et al. 2016) ? Par contre, dans deux communes du sud de la France, des femelles de $M$. sculpturalis ont été observées alors qu'elles vidaient le contenu de cellules larvaires d'osmies (Osmia bicornis (L. 1758) et $O$. cornuta (LATREILLE 1805)) pour installer leurs propres nids. De la même façon, en Autriche, une femelle a été observée vidant le contenu du nid de la guêpe (elle-même exotique) Isodontia mexicana (SAUsSuRe, 1867) (fig. 7).

\section{Impacts écologiques}

Les abeilles véhiculent une image positive associée à l'apiculture et à leur rôle dans la pollinisation, largement médiatisé ces dernières années. Aussi, en comparaison d'autres animaux ou végétaux, une moindre attention est portée aux potentiels impacts négatifs sur les écosystèmes de l'introduction d'abeilles exotiques. Pourtant, plusieurs exemples montrent que ces introductions peuvent avoir des conséquences écologiques graves. Par exemple,
Bombus dahlbomii GUERIN-MeneviLle 1835, seule espèce de bourdon naturellement présente, et endémique, en Patagonie a rapidement décliné suite à l'introduction de deux espèces de bourdons européens, Bombus ruderatus (FABRICIUS 1775) et B. terrestris (MORALES et al. 2013).

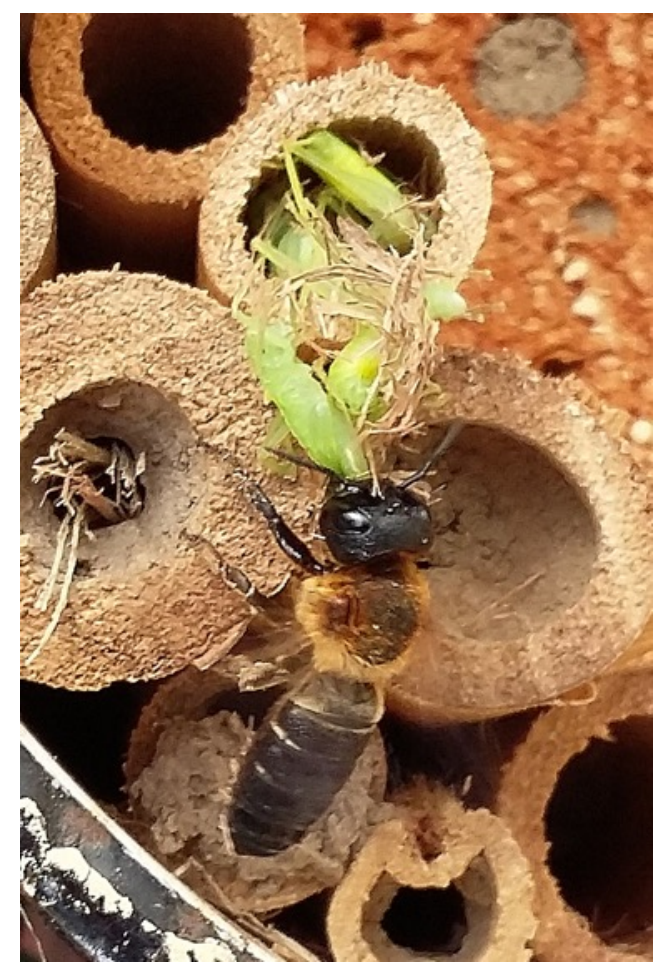

Figure 7. Femelle de Megachile sculpturalis en train de retirer d'une tige de bambou d'un hôtel à abeilles les larves d'orthoptères apportées pour sa progéniture par une femelle de la guêpe exotique Isodontia mexicana. Christian Treitner,Vienne, Autriche, 2017. 
GOULSON (2003) liste cinq types d'impacts négatifs associés à l'introduction d'abeilles exotiques : (1) la compétition pour les ressources florales avec les pollinisateurs indigènes ; (2) la compétition pour les sites de nidification ; (3) l'introduction conjointe de pathogènes ; (4) la pollinisation de plantes exotiques et (5) les effets négatifs sur la reproduction des plantes indigènes.

Nous manquons de recul aujourd'hui pour évaluer les impacts associés à la présence de $M$. sculpturalis hors de son aire d'origine. Les premières observations de terrain, en Amérique $\mathrm{du}$ Nord et en Europe, incitent cependant à porter une attention particulière à la compétition possible pour les sites de nidification entre $M$. sculpturalis et les espèces d'abeilles indigènes.

Si certains types de substrat sont en quantité limitée dans certains environnements, un phénomène de compétition peut subvenir. Par exemple, l'occupation par M. sculpturalis de cavités creusées par des xylocopes peut-elle affecter à plus ou moins long terme les populations de ces derniers ? Par ailleurs, $M$. sculpturalis a un impact direct sur la mortalité d'autres insectes en vidant les nids et les larves qui s'y trouvent. Il est difficile d'estimer la fréquence de ce type d'évènements et ses conséquences pour les populations d'insectes concernés. En Europe, certaines espèces des genres Anthidium, Lithurgus, Osmia, Xylocopa ou encore Megachile lagopoda (L., 1761) (David Genoud, com. pers.) sont des espèces susceptibles d'être négativement affectées par la compétition pour les sites de nidification ou l'usurpation de nids, dans des situations de populations fragiles ou de sites de nidification en nombre limité.

\section{L'histoire continue}

A l'instar du Frelon asiatique (Vespa velutina LEPELETIER 1836), de la Coccinelle asiatique (Harmonia axyridis (PALLAS 1773)), de la Pyrale $\mathrm{du}$ buis (Cydalima perspectalis (WALKER 1859)), $\mathrm{du}$ Longicorne asiatique (Anoplophora glabripennis (MotschULSKY 1854)) ou du Moustique tigre (Aedes albopictus (SKUSE,1894)), M. sculpturalis a rejoint la longue liste d'insectes originaires d'Asie installés en France métropolitaine. Où en est sa présence en France? Dans les zones du pays qu'elle occupe désormais, elle est fréquemment signalée dans les espaces urbains, où de nombreuses personnes circulent et sont interpelées par cette grande abeille peu discrète. Est-elle autant présente dans les zones naturelles ? Quel est le niveau d'affinité avec Sophora japonica? Se maintiendrait-elle dans des espaces où Sophora japonica (et autres espèces végétales originaires d'Asie) seraient absentes (en particulier les zones naturelles) ? Nous encourageons les observateurs à prendre des photos et à noter le maximum de détails (photographies, date, lieu, comportement, plantes visitées, substrats utilisés pour la nidification, interactions avec d'autres abeilles). Cela permettra d'approfondir les connaissances sur l'écologie de $M$. sculpturalis et de mieux évaluer ses impacts sur la faune et la flore des pays d'introduction.

\section{Remerciements}

Nous remercions toutes les personnes qui ont partagé leurs observations de Megachile sculpturalis (voir LE FÉON et al. 2018 pour la liste complète), notamment sur les forums Apoidea-Gallica et Le Monde des insectes et sur le site du Spipoll. Merci à Valérie Andrieu-Ponel, Matthieu Aubert, Philippe Dauge, Mathieu de Flores, David Genoud, Gilles Roux et Paul Westrich pour leur aide dans la collecte d'informations sur la distribution et l'écologie de l'espèce. Merci à Romain Baghi, Daniel Mathieu, Gilles Roux, Danièle Tixier-Inrep et Christian Treitner pour avoir accepté l'utilisation de leur photo. Merci à Gérard Le Goff pour l'identification du xylocope de la figure 6. Merci à Matthieu Aubert et Lise Ropars pour leur relecture. Les auteurs des fonds de carte sont Arnaud Le Nevé (fig. 3) et Eric Gaba (fig. 4).

\section{Références bibliographiques}

Amiet F, 2012. Die Blattschneiderbiene Megachile sculpturalis Smith, 1853 (Hymenoptera, Apidae) nun auch in der Schweiz. Entomo Helvetica 5 : 157 - 159.

ANdrieu-Ponel V, Ponel P, Le FÉON V, Geslin B \& Duvallet G, 2018. À propos du comportement de butinage de Megachile sculpturalis Smith, 1853, en France méditerranéenne (Nîmes et Montpellier) (Hymenoptera, Megachilidae). Bulletin de la Société entomologique de France 123 : 49 - 54.

BARThell JF, Frankie GW \& Thorp RW, 1998. Invader effects in a community of cavity nesting megachilid bees (Hymenoptera: 
Megachilidae). Environmental Entomology 27 : $240-47$.

BARThell JF, ThORP RW, 1995. Nest usurpation among females of an introduced leafcutter bee, Megachile apicalis. Southwestern Entomologist $20: 117$ - 124 .

Bartomeus I, Ascher JS, Gibbs J, DANFORTH BN, WAGNER DL, HEDTKE SM \& WiNFREE R, 2013. Historical changes in northeastern US bee pollinators related to shared ecological traits. Proceedings of the National Academy of Sciences $110: 4656$ - 4660.

BATRA SWT, 1998. Biology of the Giant Resin Bee, Megachile sculpturalis Smith, a conspicuous new immigrant in Maryland. The Maryland Naturalist $42: 1-3$.

Bortolotti L, Luthi F, Flaminio S, Bogo G, SGolastra F, 2018. First record of the Asiatic bee Megachile disjunctiformis in Europe. Bulletin of Insectology $71: 143-149$.

Dillier F-X, 2016. Eingeschleppte Asiatische Mörtelbiene Megachile sculpturalis Smith,

1853 (Hymenoptera, Apidae) erstmals nördlich der Alpen gesichtet. Entomo Helvetica $9: 153-$ 156.

Dufrêne É, Genoud D \& Bourlet P, 2016. Sur la distribution en France de Lithurgus cornutus Fabricius, 1827 (Hymenoptera Megachilidae) : apport de données récentes. Osmia $6: 16-21$.

FALK S \& LeWINGTON R, 2015. Field guide of the bees of Great Britain and Ireland. British Wildlife Field Guides. Bloomsbury.

GeSLin B \& MoRALES CL, 2015. New records reveal rapid geographic expansion of Bombus terrestris Linnaeus, 1758 (Hymenoptera: Apidae), an invasive species in Argentina. Checklist $11: 1620$.

GIBBS J \& DATHE HH, 2017. First records of Hylaeus (Paraprosopis) pictipes Nylander, 1852 (Hymenoptera: Colletidae) in North America. Check List 13 : 2116.

Goulson D, 2003. Effects of introduced bees on native ecosystems. Annual Review of Ecology, Evolution and Systematics 34 : 1-26.

HinoJoza-Diaz IA, YAÑEZ-ORdoñez O, CheN G, Perterson AT \& Engel MS, 2005. The North American invasion of the giant resin bee (Hymenoptera: Megachilidae). Journal of Hymenoptera Research $14: 69-77$.

HinOJOSA-DIAZ IA, 2008. The giant resin bee making its way west: first record in Kansas (Hymenoptera: Megachilidae). ZooKeys 1: 67 71.
KovÁCs T, 2015. Megachile sculpturalis Smith, 1853 in Hungary (Hymenoptera, Megachilidae). Folia Historico-naturalia Musei Matraensis 39 : $73-76$.

LAPORT RG \& MINCKLEY RL, 2012. Occupation of active Xylocopa virginica nests by the recently invasive Megachile sculpturalis in Upstate New York. Journal of the Kansas Entomological Society $85: 384-386$.

Le FÉon V, Aubert M, Genoud D, AndrieuPonel V, Westrich P \& Geslin B, 2018. Range expansion of the Asian native giant resin bee Megachile sculpturalis (Hymenoptera, Apoidea, Megachilidae) in France. Ecology and Evolution 8: $1534-1542$.

MANGUM WA \& BRoOKS RW, 1997. First records of Megachile (Callomegachile) sculpturalis Smith (Hymenoptera: Megachilidae) in the continental United States. Journal of the Kansas Entomological Society 70 : 140 - 142.

MACIVOR JS \& PACkER L, 2015. 'Bee hotels' as tools in native pollinator conservation: a premature verdict? PLoS ONE 10 : e0122126.

Mangum WA, Sumner S, 2003. A survey of the North American range of Megachile (Callomegachile) sculpturalis, an adventive species in North America. Journal of the Kansas Entomological Society $76: 658-662$.

MICHENER CD, 2007. The bees of the world. 2nd ed. The Johns Hopkins University Press, Baltimore.

Morales CL, Arbetman MP, Cameron SA \& AIZEN MA, 2013. Rapid ecological replacement of a native bumble bee by invasive species. Frontiers in Ecology and the Environment 11 : $529-534$.

NiETO A, ROBERTS SPM, KEMP J, RASMONT P, Kuhlmann M, García Criado M, BiEsmeiJer JC, Bogusch P, DAthe HH, De La RÚa P, DE Meulemeester $T$, Dehon $M$, Dewulf $A$, Ortiz-Sánchez FJ, Lhomme P, Pauly A, Potts SG, Praz C, QUaranta M, RAdChenko VG, Scheuchl E, SMit J, Straka J, Terzo M, TomoziI B, Window J \& Michez D, 2014. European Red List of bees. Luxembourg, Publication Office of the European Union.

Quaranta M, Sommaruga A, Balzarini P \& FELICIOLI A, 2014. A new species for the bee fauna of Italy: Megachile sculpturalis continues its colonization of Europe. Bulletin of Insectology $67: 287-293$.

Parys K, Tripodi A, Sampson B, 2015. The Giant Resin Bee, Megachile sculpturalis Smith: new distributional records for the Mid- and Gulfsouth USA. Biodiversity Data Journal 3:e6733 
Rasmont P, Devalez J, Pauly A, Michez D \& RADChENKo VG, 2017. Addition to the checklist of IUCN European wild bees (Hymenoptera: Apoidea). Annales de la Société Entomologique de France (N.S.) $53: 17$ - 32.

ROUlston T \& MALFI R, 2012. Aggressive eviction of the Eastern Carpenter Bee (Xylocopa virginica (Linnaeus)) from its nest by the Giant Resin Bee (Megachile sculpturalis Smith). Journal of the Kansas Entomological Society 85 : $387-388$.

RUSSo L, 2016. Positive and negative impacts of non-native bee species around the world. Insects $7: 69$.

Strange JP, KOCH JB, Gonzalez VH, NemelKa L \& GRISWOLd T, 2011. Global invasion by Anthidium manicatum (Linnaeus) (Hymenoptera: Megachilidae): assessing potential distribution in North America and beyond. Biological Invasions 13 : 2115 - 2133.

VEREECKEN NJ \& BARBIER E, 2009. Premières données sur la présence de l'abeille asiatique Megachile (Callomegachile) sculpturalis Smith (Hymenoptera, Megachilidae) en Europe. Osmia $3: 4-6$.

WESTRICH P, KNAPP A \& BERNEY I, 2015. Megachile sculpturalis Smith 1853 (Hymenoptera, Apidae), a new species for the bee fauna of Germany, now north of the Alps. Eucera $9: 3-10$.

Zayed A, Constantin SA, Packer L, 2007. Successful biological invasion despite a severe genetic load. PLoS ONE 2 : e868. 